\title{
Workshop on Technical Feasibility: Initial Lessons from an IFIP WG2.7 Virtual University Case Study
}

\author{
Gilbert Cockton \\ School of Computing, Engineering and Technology, The Informatics Centre, University of \\ Sunderland, PO Box 299, Sunderland, SR6 OYN, UK \\ Tel/Fax: +441915153394/2781_Email: Gilbert.Cockton@sunderland.ac.uk
}

Abstract: $\quad$ IFIP Working Group 2.7 (13.4) on User Interface Engineering has a specific interest in the software engineering of interactive systems, especially its interaction with other design activities. In 1997 and 1998, the Working Group (WG) used a Virtual University case study to track the emergence and discussion of implementation issues across a simulated development life cycle. The case study provided examples of requirements and design decisions that had to be formulated in an implementation-dependent manner, since available implementation options would constrain the viability of specified requirements or preferred design features. Only some options were technically feasible. This should be recognised when forming requirements. Technical feasibility needs to be addressed early in development.

This report summarises the results of a workshop held during the $1998 \mathrm{EHCI}$ conference. The workshop concluded that technical feasibility cannot be fully addressed unless a mature and stable IT strategy exists for the commissioning organisation. It found that simple process models provided a better framework for analysis than narrative scenarios. A framework for identifying technical issues, technical options and option inter-dependencies was developed. The workshop reinforced the frustrating nature of technical feasibility studies using current methods. There is a clear need for further in-depth research.

Key words: IT strategy, software engineering, requirements engineering, technical feasibility, scenarios, virtual university.

The original version of this chapter was revised: The copyright line was incorrect. This has been corrected. The Erratum to this chapter is available at DOI: 10.1007/978-0-387-35349-4_22 


\section{EARLY ASSESSMENT OF FEASIBILITY}

Arguments for delaying commitment and abstracting away from implementation issues have been regularly articulated within the structured and formal methods communities. The argument is that design is eased by hiding complexity to focus on the critical aspects of the design, trapping errors early in development when the cost of correcting them is still low. There is however a presumption here that neglected complexities can be trickled back into a design in a smooth process of refinement, and indeed that these complicating factors are not the critical aspects of the design!

All aspects of software development - requirements, design and implementation - have potentially equal status. Structured and formal methods originally addressed a once ubiquitous neglect of careful requirements and design formation. However, they carry with them the danger that implementation issues will not be given due weight during early development, even though studies of developers reveal a shifting focus between requirements, design and implementation during initial activities (Guindon 1992).

Given the neglect of implementation issues, it is no surprise that software developers regularly encounter feasibility problems late in development (e.g., van Vliet (1993, p.142) cites Baber (1982), which examines projects that have failed due to technical infeasibility). Idealised decisions may ignore whether a feature can be implemented for a chosen platform (hardware, communications, system software, development tools). Current development methods provide insufficient direction on how to avoid implementation crises. They could be extended by older systems analysis approaches to feasibility studies (although relatively few feasibility studies do result in projects being cancelled!) Yet interestingly, a standard Software Engineering text only offers a simple description of a feasibility study:

An estimate is made of whether the identified user needs may be satisfied using current software and hardware technologies. The study will decide if the proposed system will be cost-effective from a business point of view and if it can be developed given existing budgetary constraints. A feasibility study should be relatively quick and cheap. The result should inform the decision of whether to go ahead with a more detailed analysis (Sommerville 1995, p. 67)

This description is incompatible with a seasoned practitioner's view:

The functional specification stage is the earliest (in life cycle terms) at which ... feasibility questions - technical and economic - can be answered with any degree of confidence, in general (Macro 1990, p. 175)

The difficulty of technical feasibility is noted in another standard text, which in turn contradicts the expert opinion above:

Technical feasibility is frequently the most difficult area to assess at this [requirements] stage of the system development process. Because objectives, functions and performance are somewhat hazy, anything seems possible if the right assumptions are made. It is essential that the process of [requirements] 
analysis and definition be conducted in parallel with an assessment of technical feasibility (Pressman 1992, pp. 148-49)

So, three experts offer three different opinions on when technical feasibility should be assessed: before any formal requirements analysis (Sommerville), alongside formal requirements analysis and definition (Pressman), alongside functional specification (Macro, but with an first feasibility study during concept development, p. 106). This confusion is compounded by the view that "the matter of major concern ... is the economic feasibility" (Macro 1990 p.185). Furthermore, anecdotal evidence suggests that current feasibility studies tend to focus almost exclusively on business feasibility. Thus commercial structured methods give far more attention to business and IT strategy than to technical issues, for example:

A Feasibility Study is a short assessment of an information system to determine whether the system can meet the specified business requirements of the organisation, and whether a business case exists for developing such a system.

... The SSADM techniques primarily assist the identification of information requirements and the assessment of technical feasibility. Feasibility activities do not describe the other aspects of feasibility in detail, ...

The current and required environments are studied and only in sufficient detail to enable a Problem Definition Statement to be developed ... and for Business System Options and Technical System Options to be identified.

\section{Objectives}

- To establish whether a proposed information system meet the specified business requirements of the organisation.

- To establish the business case for the proposed system and enable the project board to decide whether to commit resources to a more detailed study.

- To determine whether to proceed in a different direction than that envisaged in the IS [IT] Strategy.

- To enable the project board to select from a range of business and technical options and to identify the necessary to implement the chosen option.

(Sandhill 1998)

Technical issues are given limited attention in this process, and much is left to the role of Specialist Advisor, who is (Sandhill 1998)

responsible for providing input to a Feasibility Study on specific areas concern to the Study, e.g., technical feasibility, security specialists, database designers.

From the above it is clear that there is no consensus on:

a) When to carry out technical feasibility studies

b) How many such studies to carry out

c) Their relative importance in relation to economic/business feasibility studies

This is of little help to a software project team who want to address technical feasibility as early and effectively as possible. There is a pressing need to address 
technical feasibility early and systematically in the development process. Realistic software development methods must support opportunistic shifts in focus and should not obstruct opportunities to explore key implementation scenarios during requirements and design.

The software development lifecycle can be structured in many ways (Royce 1970, Boehm 1988, Gram and Cockton 1996) with respect to the number and nature of development phases and the relationships between them. However, all idealised development lifecycles begin with problems to be addressed and end with operational software, which has an internal structure, or software architecture. This architecture comprises a set of components and a set of links between them A software architecture represents an allocation of function to structure (Kazman et al. 1994), in that separate functions are achieved by individual components or some linked combination. Software developers rarely have freedom of choice in the design of software architectures, as they can rarely provide optimal support for all requirements. Compromises and trade-offs are generally unavoidable.

IFIP Working Group 2.7 (WG 2.7) sought to develop an understanding of implementation-dependencies during development by following through a case study. One aim of the case study was to explore the identification and formation of compromises and trade-offs The EHCI'98 workshop reported here concludes this case study by addressing how technical feasibility could be studied early in development.

\section{THE VIRTUAL UNIVERSITY AS A CASE STUDY}

The topic of the case study was the Virtual University. Unlike traditional universities, the activities for a virtual university are not restricted to fixed times in fixed places. Instead, activities can be distributed in space and/or time by combining the storage and editing facilities of computer systems with the multimedia communication capabilities of broadband digital networks.

The systems architecture of a Virtual University (VU) is critical to its success. The functions of the university must be allocated to appropriate software and hardware components, which in turn must be linked by appropriate communication channels. In addition, this architecture problem for a VU has to be addressed at least three levels (individual, group and corporate).

This section outlines work at three WG2.7 meetings before the EHCI 98 workshop. Notes on the results of each meeting are reported elsewhere (Cockton 1998). The first meeting focussed on requirements, and was held in combination with some members of IFIP WG2.9 (Requirements Engineering) at Stone Mountain Inn, Georgia, USA (20-22nd March 1997). The second meeting at Certosa di Pontignano, Italy (13-15th September 1997) focussed on design and was attended by the chair of IFIP WG13.2 (User Interface Design). The third meeting focussed on implementation and was held at ISI, California, USA (16-18th April 1998). Most members of WG2.7 attended at least one meeting. Observers and guests also contributed to working group discussions (see Cockton 1998 for lists of contributors). 
With between 1.5 and 2.5 days work on the case study at each WG meeting, there was little time for tutorial material on specific methods and techniques. Further complications arose from the fact that all methods and techniques need to be tailored to the context of development, and it was not clear how to tailor for the unusual context of WG2.7!

Existing methods were used at the ISI meeting (see Cockton 1998), when WG members used methods and approaches that were familiar to them, although a novel approach to scenarios emerged in the process. Rather than vary the human scenario details of who, what, where, when and why, technical variation points were altered within the same core scenario. This appeared to offer a way to address technical feasibility early in software development. A core scenario forms the basis for initial discussions. Implementation issues associated with the scenario can then be discussed, and the effects of selected technical variations (e.g., $28 \mathrm{~K}$ line) and some social changes (e.g., no late joiners, fewer/more questions) can also be explored.

The scenarios in this new approach are called Technical Envisionment Scenarios (TEnS). TEnS could become a useful tool for feasibility studies by increasing the chances of revealing implementation difficulties. To avoid missing some critical technical variation points (e.g., issues known from the European Virtual Summer School were missed during the Spring 1997 requirements meeting - Cockton 1998), focused prototyping needs to be carried out to complement scenario-based analyses. Where prototypes cannot resolve uncertainties, a project should almost certainly be abandoned. However, technical prototypes "often take years to do, and cost millions - in any currency - before they demonstrate their virtues" (Macro 1990 p.186). There is clearly a challenge in focusing the prototyping effort. One possible approach is to simulate software architectures.

Some argue that architecture should be examined as part of the requirements process (e.g., McDermid 1994). It is not clear from our case study that this is so. Software architecture made no contribution during the exercise. Implementation constraints are certainly central to the analysis of technical feasibility, but they tend to arise in a piecemeal manner rather than arising from complete and coherent architectural models. If TEnS and prototyping are sufficient for feasibility studies, then software architectures have no separate role in early technical feasibility. Choices of concrete architecture, especially for component integration and interoperation will arise as simple technical variation points. The role and value of software architectures needs to be further explored to establish whether it does have any role in early technical feasibility studies. This matches the findings of the SAMSA project, which explored architecture simulation for feasibility analysis. SAMSA identified further research issues rather than developed proven architecture based approaches to technical feasibility (Boehm and Scaachi 1996).

\section{THE EHCI'98 WORKSHOP}

Technical feasibility is an important issue. The approach taken by WG2.7 overlaps with recent leading work on 'buildability'. This section reports the framework developed within the EHCI 98 workshop. 


\subsection{The Plan}

A range of issues had been identified for the workshop:

- Where are good technical feasibility (TF) methods documented? Are there best industrial practices that could be formalised?

- Are Technical Envisionment Scenarios well defined as a technique? Are they credible? What needs to be done to improve them?

- How should TF studies (i) present and (ii) defend conclusions?

- What, if anything, is the role for architectural analysis in a TF studies?

- How does prototyping fit into TF studies?

- Can methods from requirements engineering be simplified for rapid use in the early stages of feasibility analysis (which? how?)

- How can TFS influence further development (e.g., stop it, phase it, scope the initial functionality, or provide input to later development stages)?

- Can previously published research be used to defend TF decisions?

\subsection{The Reality}

Only the first two issues were explored, with most of the emphasis on the second. On the first issue, Len Bass reported that scenario-based approaches to technical feasibility and similar issues (e.g., security) were used on SEI projects. Although the third issue was not addressed, there are useful structures for feasibility reports (e.g., Pressman 1992, p.150).

At the first session Technical Envisionment Scenarios (TEnS) were presented, using the remote synchronous lecture example from the ISI meeting (Cockton 1998). A new example, based on a student enrolment scenario, was developed at the first workshop session. The aims were to establish how well TEnS were defined as a technique, whether they were credible and what needed to be done to improve them.

It soon became apparent that the narrative format used for TEnS at the ISI meeting was too detailed. Simple process models were a better starting point. Thus the main activities within the enrolment process were identified as:

1. Student: Expressing an interest in a course

2. Student: Supplying information about oneself

3. Student, Advisor: Discussing possible options and identifying best choices

4. Student: Making final choice of course

5. Student: Paying for course.

We could see different ways of composing these activities within a temporal framework. For example, would a student pay (4) before making a final choice (3)? Would expressing an interest (1) and supplying full relevant information (2) be a pre-requisite for proceeding to discussing possible options and identifying best choices (3)? How long could a student spend on discussion before being forced to make up their mind (the 'permanent student' could be replaced by even more frustrating 'permanent enrolee')? Such uncertainties exposed a point that would dominate the workshop.

Policy decisions must be in place before technical feasibility can be assessed 344 
This is consistent with the model for feasibility studies within SSADM (Sandhill 1998) where an IT strategy has to be in place before feasibility can be assessed. However, many of the necessary policy decisions only arose because technical issues were under consideration. This would suggest that technical envisionment should be used to assess the stability of an IT strategy. A stable IT strategy would thus be defined as one that would not require revision when any previously unaddressed technical issues are examined. To some extent, this addresses the issue of when technical feasibility can be assessed. If system's development occurs within the context of an organisation's IT/IS strategy, then technical feasibility can be addressed before requirements definition (Sommerville's preference). When no stable strategy exists, Macro's preference for feasibility studies alongside outline system design seems to be more realistic.

It was possible to continue the analysis in the absence of Virtual University policies on time limits on enrolment processes and payment points (Pay for a year? Pay for a module? Pay for a module without committing to a course ${ }^{\dagger}$ ?) For each activity it was possible to identify a set of technical/strategy concerns as follows:

1. Expressing interest: fixed set of programmes or free student choice of modules? Full or part programmes (individual modules)?

2. Supplying information: authentication (use trusted third parties such as national examination boards?), hardware/software needs (digital photos? security mechanisms, private keys?)

3. Discussing options: format for course requirements, ability to automatically check pre-requisites, evidence/audit of student qualifications, special cases, timing (synchronous/asynchronous, fixed times in year?), modalities (phone, video, email), agent (single human, help-desk team, software agent), availability of agent ( 24 hours a day 7 days a week?), impact of and information about a course's schedule, access to previous and current students as part of an applicant's 'due diligence'

4. Making final choice of course: how does the student 'sign'? What is the nature of the contract? What about later changes (is a course change a re-enrolment?)

5. Paying: different costs of courses? Module costs for flexible and partial courses? Identification of student? Fund transfer? Receipts? Relationship to contract issues? Manual system as an alternative? Currency?

Technical options and policy decisions are inseparable. The level of requirement for secure payments and authentication depends on the Virtual University's policies e.g., payment schedules, contract details, course structure and flexibility, costing models, policy on pre-requisites. It was thus not possible to explore technical issues further unless we committed to specific policies. When developing TEnS, we had hoped to accommodate technical and policy variations simultaneously, but this quickly proved to be too ambitious. A clear problem statement is essential. Note however that for one existing structured method, the aim of a feasibility study is to allow "a Problem Definition Statement to be developed" (Sandhill 1998). Our experience is that technical feasibility requires a clear definition of organisational

† Note that British terminology is used above: a Course spans several years and leads to a degree (programme in North America). Courses are composed from modules in the UK (courses in North America!) Many European countries do not have modular courses. A course of study is often fixed with limited choice. 
policy in order to proceed. Thus a feasibility study should begin with one form of problem statement (organisational policies and IT strategy), and end with a specific problem statement that scopes out the technology to be developed and the technical options that are acceptable and/or mandatory for a viable system.

One interesting example that clarifies the role of organisational policy was enrolment onto high demand courses. This gave rise to several questions:

- Will students and advisors be aware of courses that are 'filling up'?

- How and why can courses at a Virtual University 'fill up'? Do enrolments for parts of courses contribute to filling up? How?

- Do current manual systems cope well enough? Will they scale for higher student targets and lower course budgets?

- Would enrolment operate on a 'first-come first-served' basis for suitably qualified candidates (as in UK and USA)? If so, what would 'first come' mean? Earlier time of sending choice message? Earlier arrival at system of choice message? Any compensation for network delays and system failures (students', university's and intermediaries') or compensation for time-zone advantages? Process in single transactions or batch (when?)? When/how to confirm a place on a 'full' course ...

- Would enrolment be cut-off at a fixed time and then a ballot decide who would be given a place on a course (as in Denmark)?

Technical feasibility for managing courses that were 'filling up' could not be explored until these related policy issues were resolved. Similar issues arise for courses that are not 'full enough'? Do courses have to break even? Is there a business model for each course? At what point does a virtual university enter into a contract to run a course?

For the remainder of the workshop, we assumed that policy decisions and a stable IT strategy existed for a Virtual University. This strategy would commit to the use of IT or (part-)manual solutions for different activities (e.g., manual payments system, automatic advice agents). Technical options could then be identified. These options would not be orthogonal, since the choice of some options would entail or rule out some options for other choices. Options should thus be considered in groups before examining technical issues such as reliability in detail.

\subsection{An Emerging Framework}

The recognition of the need for mature and stable IT strategies (and in turn their dependence on some technical envisionment) was a key important insight at this stage. TEnS were thus quickly replaced by a new approach as follows:

1. Identify the main processes within an application domain, e.g., for a Virtual University - present lecture, enrol student ...

2. Iterate through steps 3 to 9 below for each process, merging outcomes into a single feasibility plan and noting needs for/securing necessary policy/strategy decisions/amendments.

3. Select a process within a Virtual University

4. Decompose the process into activities and identify temporal dependencies

5. Identify areas of technical concern (e.g., queuing for popular courses)

6. Identify values for requirements associated with each area 
7. Isolate technical options for each activity

8. Group options on the basis of dependencies (option A for activity $\mathrm{X}$ requires option B for activity $\mathrm{Y}$ - or for some generic system property, e.g., security features such as digital watermarking of content may require high performance processors). Where possible, associate clusters of options with current implemented IT solutions as reference points.

9. Specify required expert technical studies or consultations.

The output of this procedure is thus a set of questions and identification of experts and/or studies that can answer them adequately. No example was developed in any depth beyond Step 4 above. Indeed, the above procedure was never followed through systematically. The workshop tended to gravitate towards brainstorming, and the actual relevance of an observation to a particular step of the procedure was often reverse-engineered on an ad-hoc basis. Thus for Steps 5 to 7 for enrolment, areas of technical concern included student authentication, accessibility and role of public enrolment statistics for popular courses, timing and time scales for specific activities (especially consultation and choice activities). These concerns gave rise to requirements for security, capacity, availability and performance, amongst others (again, we did not have time for thorough derivations). Options that could address technical concerns within the constraints of recognised requirements included a manual enrolment system, lotteries or complex queuing systems for popular courses, access to advisers via phone, email or videophone, appointments systems for consultations, and call-centres for consultations on demand.

The above framework was used to quickly re-analyse the remote lecture scenario from the ISI meeting. More of the framework was applied here than to the enrolment example (we were learning quickly!). The lecture process was decomposed into activities such as setting up (considered at the 1997 design meeting), starting the lecture, accommodating late joiners, taking and answering questions, requesting and responding to votes, conversations between students, and gauging the mood of the audience. Late joining was the main area of technical concern, followed by protecting property rights for digital content (see 4.2 below for further examples). For IS/IT strategy, some constraints for Step 6 had already been considered (requiring a specific web browser, restricting the use of pluggable applications to ones approved and supplied by the university). Further requirements could be expressed with reference to values for security, adaptability, performance and capacity, amongst others. Options that could address technical concerns within the constraints of recognised requirements video/audio buffering, slow scan video, stills-only, audio-only, digital watermarks and encryption.

\section{TOWARDS A METHODOLOGY}

In the time available (and in the absence of a virtual vice-chancellor, president or principal!), we could not complete a methodology for technical feasibility. This was not surprising. The problem is pressing and has received sustained attention from highly experienced groupings of IT experts. Nevertheless, we feel that our humancentred approach based on organisational processes does provide a rapid way into an analysis of technical feasibility. In particular, the framework above could be used to 
'shake down' an IT strategy. Consideration of processes and issues would continue until the strategy stabilised. After that, more detailed technical studies could proceed, perhaps using architectural analysis (e.g., Boehm and Scaachi 1996).

We hope that others can build on the insights we developed during the workshop. At the least, we hope that they confirm the thinking of other groups who have addressed technical feasibility. In addition, we have adopted some initial positions on some issues and hope that these too will be useful.

\subsection{What is Technical Feasibility?}

Technical feasibility is 'buildability'. It has to be demonstrated (but without actually building the system!) It is demonstrated by specifying a coherent set of technical options that are viable within known and mandated resources and requirements. These options should cover all areas of technical concern.

A proposed IT solution is technically infeasible when there is no possible option for a key feature, or no option that meets all specified constraints.

\subsection{What Are Areas of Technical Concern?}

Areas of technical concern are aspects of activities that cannot be addressed by basic implementation strategies, i.e., there is no stable solution, as e.g., for basic document editing (i.e., use off-the-shelf wordprocessor).

In the case study, some further areas of concern for the remote synchronous lecture were (see Cockton 1998 for more details):

- Functionality and inter-operability for pluggable applications, security for unauthorised applications

- Operability for instructor (avoid high cognitive load during lecture)

- Security of content and transmission

- Functionality of history/playback/re-wind mechanisms

- Accessibility for students

- Observability of network state

\subsection{Why do Technical Concerns Arise?}

Areas of technical concern arise because a requirement associated with an activity has a value that cannot be obviously met by current known options. As well as the requirements mentioned in 3.3 and 4.2 above, further requirements include maintainability, operability and modifiability

Values for these requirements must be set in accordance with IT strategy and related organisational policies.

\subsection{How do Requirements Guide Feasibility Studies?}

High level requirements allow possible options to be identified once values and tolerances (allowable flexibility) are set for each of them (even if only in outline). 
These options are then grouped according to the framework developed in Section 3.1. These structures and associated requirements form the basis for commissioning specialist studies or opinions from technical experts.

\section{CONCLUSIONS}

The work reported here will hopefully be useful to future research on technical feasibility. In particular, the following positions appear to be robust and should form a reliable basis for further work:

- Mature IT strategies and relevant organisational policies must be in place for technical feasibility studies to proceed effectively.

- Opportunistic considerations of technical feasibility are a useful approach to checking the stability of an IT strategy and relevant organisational policies

- Technical feasibility has to be re-visited in different ways at different points of the development process, especially during strategy formation, requirements analysis and architectural analysis

- Processes of component activities provide a lightweight approach to identifying areas of technical concern and associated requirements

- Options need to be related in terms of dependencies and incompatibilities before commissioning specialist studies and opinions

- The output of an initial feasibility study should be a plan for commissioning specialist studies and opinions, which form the basis on which a final technical feasibility report can be based

Realistic tests of approaches to technical feasibility can only be carried out on real projects with real organisational policies, real IT strategies and real technical experts and studies. Technical feasibility cannot be effectively addressed in the abstract. However, brainstorming, envisionment and role playing activities such as those used during the WG2.7 case study are valuable in exposing the main activities and inter-dependencies in a structured approach to technical feasibility.

\section{ACKNOWLEDGMENTS}

Gilbert Cockton's participation in the first two WG meetings on the case study was funded by UK EPSRC grant GR/K82727. Workshop participants worked effectively over the four workshop sessions at EHCI98. I would like to thank them for their contributions to the work reported here. The participants were:

- Margherita Antona, FORTH - Institute of Computer Science, Crete, Greece.

- Len Bass, Software Engineering Institute, Carnegie-Mellon University, USA

- Michael Freed, NASA Ames Research Centre, USA.

- Christian Gram, Technical University of Denmark.

- Dimitris Grammenos, FORTH - Institute of Computer Science, Crete, Greece.

- Xiangshi Ren, Tokyo Denki University, Japan

- Chris Roast, Sheffield-Hallam University, UK. 
- Helmut Stiegler, STI-Consulting Gmbh, Munich, Germany.

\section{REFERENCES}

Baber R. (1982), Software Reflected, North-Holland.

Boehm, B.W.. (1988), 'A spiral model of software development and enhancement', COMPUTER, 21(5): 61-72.

Boehm, B.W. and W. Scacchi, (1996) Simulation and Modeling for Software Acquistion (SAMSA): Air Force Opportunities (Extended Report), http://sunset.usc.edu/SAMSA/samcover.html, Created March 1996, Accessed 28/10/98.

Cockton, G. (1998), IFIP Virtual University Case Study, http://osiris.sunderland.ac.uk/ cs0gco/IFIP/ifip_index.htm, Created August 1998

Gram, C. and G. Cockton (eds.) (1996), Design Principles for Interactive Systems, Chapman and Hall, 1996.

Guindon, R. (1992), 'Requirements and Design of DesignVision, An Object-Oriented Graphical Interface to an Intelligent Design Assistant', Proc. CHI'92, 499-506.

Kazman, R., L. Bass, G. Abowd and M. Webb (1994), 'SAAM: A method for analyzing the properties of user interface software architectures', Proc. ICSE-16, Sorrento, Italy, 81-90.

McDermid, J. (1994). "Requirements Analysis: Orthodoxy, Fundamentalism and Heresy", in Jirotka and Goguen (eds.), Requirements Engineering: Social and Technical Issues, Academic Press, London

Macro, A. (1990), Software Engineering: Concepts and Management, Prentice-Hall

Pressman, R. S. (1992), Software Engineering: A Practitioner's Approach, 3rd edition, McGraw-Hill.

Royce, W.W. (1970), 'Managing the development of large software systems', Proc. WESTCON, Ca., USA.

Sandhill Consultants Ltd, (1998) Stage: Feasibility Study (Feasibility Study Module), http://www.sandhill.co.uk/pware/feas/ac06190.htm, Created 1/7/98, Accessed 28/10/98.

Sommerville, I. (1995), Software Engineering, 5th edition, Addison-Wesley.

Van Vliet, H. (1993), Software Engineering: Principles and Practice, Wiley.

\section{BIOGRAPHY}

Professor Gilbert Cockton FRSA, is Research Chair in Human-Computer Interaction in the School of Computing and Information Systems at the University of Sunderland in North-East England. During 15 years of HCI research, he has (co-) authored nearly 60 publications on several topics. He became a member of WG2.7 in 1988 and secretary in 1993. He co-edited WG2.7's monograph on interactive systems development with Christian Gram (Gram and Cockton 1996). His interest in early assessment of technical feasibility arises from seven years of commercial and government consultancy, including independent design and development work and as an expert project monitor and proposal evaluator for the European Commission. A Fellow of the Royal Society for Arts, Manufacture and Commerce, his user interface work has been exhibited at a Scottish design exhibition. 